ANNALES

POLONICI MATHEMATICI

$86.2(2005)$

\title{
Critique of "Two-dimensional examples of rank-one convex functions that are not quasiconvex" by M. K. Benaouda and J. J. Telega
}

\author{
by Patrizio NefF (Darmstadt)
}

\begin{abstract}
It is noted that the examples provided in the paper "Two-dimensional examples of rank-one convex functions that are not quasiconvex" by M. K. Benaouda and J. J. Telega, Ann. Polon. Math. 73 (2000), 291-295, contain unrecoverable errors.
\end{abstract}

1. Introduction. We consider variational integrals

$$
I(\varphi)=\int_{\Omega} W(\nabla \varphi(x)) d V,
$$

defined for sufficiently regular deformations $\varphi: \Omega \subset \mathbb{R}^{m} \rightarrow \mathbb{R}^{n}$ where $\Omega$ is a bounded open subset of $\mathbb{R}^{m}$. Here, $\nabla \varphi(x)$ denotes the deformation gradient at $x \in \mathbb{R}^{m}$ and $W$ is a continuous function on the space $\mathbb{M}^{m \times n}$ of all real $m \times n$ matrices. One of the important problems in the calculus of variations is to characterise the integrand $W$ for which the integral $I$ is lower semicontinuous. In this respect the following notions have been introduced (see e.g. $[1-3,5,6])$ :

1. $W$ is rank-one convex if for each matrix $F \in \mathbb{M}^{m \times n}$ and each rank-one matrix $B \in \mathbb{M}^{m \times n}$, the real-valued function $t \mapsto W(F+t B)$ is convex.

2. $W$ is quasiconvex if for any matrix $F \in \mathbb{M}^{m \times n}$,

$$
\begin{aligned}
\forall \psi: \mathbb{R}^{m} \rightarrow \mathbb{R}^{n}, \psi(x)=F . x, x \in \partial \Omega: \\
\qquad \int_{\Omega} W(\nabla \psi(x)) d V \geq W(F) \cdot|\Omega| .
\end{aligned}
$$

Quasiconvexity implies that the homogeneous deformation $\varphi(x)=F . x$ is energy optimal for homogeneous boundary conditions.

For "nice" integrands $W$ the quasiconvexity condition is necessary and sufficient for the weak lower semicontinuity of $I$. However, quasiconvexity is

2000 Mathematics Subject Classification: 49J40, 49J45.

Key words and phrases: quasiconvex, rank-one convex. 
a nonlocal condition, difficult to check in practice. The rank-one convexity is in principle easy to verify: for $W \in C^{2}$ it is the Legendre-Hadamard ellipticity condition. Moreover, it can be shown that quasiconvexity implies rank-one convexity. The converse is not true as has been shown by Šverák [8] for the case $m \geq 2$ and $n \geq 3$. It is a long standing open problem [7] whether for $m=n=2$ rank-one convexity implies quasiconvexity.

The authors of [4] claim to have found a counterexample for this twodimensional case. I show that their example is not a counterexample.

2. Analysis. In the following, let $\mathbb{M}^{2 \times 2}$ denote the set of two times two matrices and define for the deformation $\varphi: \mathbb{R}^{2} \rightarrow \mathbb{R}^{2}$ the corresponding deformation gradient

$\nabla \varphi\left(x_{1}, x_{2}\right)=\left(\begin{array}{ll}\varphi_{1, x_{1}}\left(x_{1}, x_{2}\right) & \varphi_{1, x_{2}}\left(x_{1}, x_{2}\right) \\ \varphi_{2, x_{1}}\left(x_{1}, x_{2}\right) & \varphi_{2, x_{2}}\left(x_{1}, x_{2}\right)\end{array}\right)=\left(\begin{array}{ll}F_{11}\left(x_{1}, x_{2}\right) & F_{12}\left(x_{1}, x_{2}\right) \\ F_{21}\left(x_{1}, x_{2}\right) & F_{22}\left(x_{1}, x_{2}\right)\end{array}\right)$.

In $\left[4\right.$, Lem. 4.1] it is claimed that the quadratic function $W: \mathbb{M}^{2 \times 2} \rightarrow \mathbb{R}$ (the function $g$ there) given by

$$
W(F)=F_{11} F_{22}+F_{12}^{2}+F_{21}^{2}
$$

is rank-one convex and in $[4$, Lem. 4.2$]$ it is argued that $W$ is not quasiconvex.

Let us rewrite $W$ in the form

$$
\begin{aligned}
W(F) & =F_{11} F_{22}+F_{12}^{2}+F_{21}^{2} \\
& =F_{11} F_{22}-F_{12} F_{21}+F_{12}^{2}+F_{21}^{2}+F_{12} F_{21} \\
& =\operatorname{det} F+\left(F_{12}^{2}+F_{21}^{2}+F_{12} F_{21}\right) .
\end{aligned}
$$

By Young's inequality it is easy to see that

$$
\forall F \in \mathbb{M}^{2 \times 2}: \quad F_{12}^{2}+F_{21}^{2}+F_{12} F_{21}>0 .
$$

Therefore, $F \mapsto F_{12}^{2}+F_{21}^{2}+F_{12} F_{21}$ is a strictly positive quadratic form, hence strictly convex. Altogether, $W$ is the sum of the quasi-affine function $F \mapsto$ $\operatorname{det} F$ and a strictly convex term, hence rank-one convex and quasiconvex. The error in [4, Lem. 4.2] stems from the fact that the test-function used is indeed not periodic on the unit cube $[0,1]^{2}$.

In $[4$, Th. 3.1] the same error occurs. The test-function is again not periodic on $[0,1]^{2}$, therefore, Theorem 3.1 is wrong. Moreover, the meaning of Theorem 3.1 as far as a counterexample to the above mentioned open question is concerned, is not clear to the author, since "rank-one convexity at 0 " does not imply rank one convexity.

The observation that this paper is erroneous is not new; indeed, it is clearly pointed out by Baisheng Yan in Mathematical Reviews [MR1785693 (2001g: 49005)]: 
"The authors claim to provide two-dimensional examples of rank-one convex functions that are not quasiconvex. \{Reviewer's remarks: Theorem 3.1 and Lemma 4.2 appear to be incorrect because Lemma 2.4 is wrongly quoted and used. The periodic functions in Lemma 2.4 should be of $[0,1]^{n_{-}}$ period, as originally stated in the papers $[4,8]$ cited [B. Dacorogna, Direct methods in the calculus of variations, Springer, Berlin, 1989; MR0990890 (90e:49001); V. Šverák, Proc. Roy. Soc. Edinburgh Sect. A 120 (1992), no. 1-2, 185-189; MR1149994 (93b:49026)]. This paper had the good intention to solve a very hard open problem, but unfortunately appears not to contain any result that is new and correct.\}"

Acknowledgements. The present author was kindly made aware by the unknown referee of the already existing Mathematical Reviews discussion by Baisheng Yan.

\section{References}

[1] J. M. Ball, Constitutive inequalities and existence theorems in nonlinear elastostatics, in: R. J. Knops (ed.), Nonlinear Analysis and Mechanics: Heriot-Watt Symposium, Vol. 1, Pitman, London, 1977, 187-238.

[2] - Convexity conditions and existence theorems in nonlinear elasticity, Arch. Rat. Mech. Anal. 63 (1977), 337-403.

[3] J. M. Ball and F. Murat, $W^{1, p}$-quasiconvexity and variational problems for multiple integrals, J. Funct. Anal. 58 (1984), 225-253.

[4] M. K. Benaouda and J. J. Telega, Two-dimensional examples of rank-one convex functions that are not quasiconvex, Ann. Polon. Math. 73 (2000), 291-295.

[5] P. G. Ciarlet, Three-Dimensional Elasticity, Stud. Math. Appl. 1, Elsevier, Amsterdam, 1988.

[6] B. Dacorogna, Direct Methods in the Calculus of Variations, Appl. Math. Sci. 78, Springer, Berlin, 1989.

[7] P. Pedregal and V. Šverák, A note on quasiconvexity and rank-one convexity for $2 \times 2$ matrices, J. Convex Anal. 5 (1998), 107-117.

[8] V. Šverák, Rank-one convexity does not imply quasiconvexity, Proc. Roy. Soc. Edinburgh Sect. A 120 (1992), 185-189.

AG6, Fachbereich Mathematik

Darmstadt University of Technology

Schlossgartenstrasse 7

64289 Darmstadt, Germany

E-mail: neff@mathematik.tu-darmstadt.de 